\section{THE HEART IN RHEUMATOID ARTHRITIS}

\section{BY}

\section{ALFRED S. ROGEN, M.D., F.R.F.P.S.G.}

(From the Department of Materia Medica and Therapeutics, Glasgow University, and the University Medical Clinic, Stobhill Hospital)

Rheumatic fever and rheumatoid arthritis are generally regarded as distinct diseases which have much in common, but the possibility of their having a common aetiology has often been discussed. As cardiac lesions are characteristic of rheumatic fever, many workers have sought similar lesions in rheumatoid arthritis. Recently tissues obtained at necropsy from patients who had suffered from rheumatoid arthritis have been studied, and a high incidence of heart lesions has been reported showing histological characteristics similar to those of rheumatic fever.

Only the more recent published work will be reviewed here. Master and Jaffe (1932) reported on the electrocardiographic findings in 17 patients with rheumatoid arthritis. The absence of abnormalities - as contrasted with a $100 \%$ incidence of electrocardiographic changes in rheumatic fever-prompted them to suggest that when in doubt as to the differential diagnosis between these two conditions a normal electrocardiogram was in favour of a diagnosis of rheumatoid arthritis ; but the number of patients was so small that this suggestion must remain a tentative one. Dawson and Tyson (1936) presented evidence to show that the two diseases are intimately related. Their similarity was stressed under the headings of familial relationship, geographical distribution, initiating factors, seasonal incidence, age incidence and clinical manifestations of the two diseases in different age periods, pathological similarities, and immunological findings. In a series of 100 patients they found 7 with unequivocal signs of rheumatic heart disease ; the onset of disease in all these patients occurred most commonly in the second and third decades of life. Collins (1937) and Bennett, Zeller, and Bauer (1940) reported that the nodules of rheumatoid arthritis and rheumatic fever were not similar on microscopical examination. On the other hand, Baggenstoss and Rosenberg (1941) quote various workers who reported the histological similarity of the subcutaneous nodules of the two diseases. Baggenstoss and Rosenberg (1944) described two cases of rheumatoid arthritis with cardiac lesions very similar histologically to the subcutaneous nodules present in that disease. They state further that, as a rule, the cardiac lesions of rheumatoid arthritis are indistinguishable from those produced by rheumatic fever. They had previously (1941) found the rheumatic type of heart disease in 14 of 25 cases of rheumatoid arthritis which came to necropsy; in only 7 of these 14 had there been any clinical evidence of heart disease. In 6 the mitral valve was involved, and in 2 of these there was advanced stenosis. Bayles (1943) in 23 necropsies on subjects with rheumatoid arthritis found 6 cardiac lesions suggestive of rheumatic fever, 1 possibly active, and 5 inactive. Young and Schwedel (1944) in a post-mortem study of 38 cases of chronic rheumatoid arthritis found cardiac lesions in 33 , in 25 of which given. It was further pointed out that microscopical examination of the myocardium showed Aschoff bodies. in one case only, but that this was not surprising as the majority of the patients were old at death. Again, scarring in the myocardium occurring in only two instances was considered to be an unduly low incidence for acute rheumatism. The annotator also made the important observation that "in rather a large proportion of cases clinical examination had given negative results." This apparent discrepancy between the clinical assessment and the post-mortem findings calls for careful consideration. It suggests that valvular disease may occur in rheumatic fever without producing clinical signs, in which case one could never be sure that there was no valve damage. In other words, the lesions might be demonstrable histologically and yet fall short of interfering with the valve mechanisms. Alternatively, there might be some other fundamental difference between the valvular lesions of the two diseases, so that those of rheumatic fever resulted in the production of murmurs while those of rheumatoid arthritis did not-a state of affairs which is highly improbable. Fraser (1945) noted a 14\% incidence of valvular disease of the heart on clinical examination in 110 patients suffering from rheumatoid arthritis. This was merely an incidental finding, however, and the valvular lesions are not classified. Paul D. White (1944) states that, in rheumatoid arthritis, pericarditis and endocarditis and myocarditis may infrequently appear as complications not to be ascribed, at least in all cases, to a coincident rheumatic fever.

\section{The Present Investigation}

The object of this paper is to record the results of a detailed examination of the cardiovascular system in 33 consecutive cases of rheumatoid arthritis admitted to municipal hospitals in Glasgow. The severity of the illness in these patients is given in accordance with the classification suggested by Sclater (1943). Of the 33 patients 4 fell into Group I, 10 into Group II, 11 into Group III, and 8 into Group IV. Besides the usual clinical examination, every patient was submitted to electrocardiographic investigation. In addition, radiological examinations of the heart were made in the earlier patients of the series, but this was soon found to be impracticable because of technical difficulties associated with the gross deformities of the joints resulting in inability to get many of the patients into the suitable position. None gave a previous history of rheumatic fever.

Eleven of the patients were males and 22 were females. The age distribution is shown in Tables I and II, which also give the duration of the illness in each case.

TABLE I.-Age Incidence: Males

\begin{tabular}{l|c|c|c|c|c|c|c}
\hline & \multicolumn{8}{|c}{ Age Groups } \\
\hline $\begin{array}{c}\text { No. in each group } \\
\text { Duration of illiness } \\
\text { (in years) of } \\
\text { each patient }\end{array}$ & $1,4,2$ & $26-50$ & $51-55$ & $56-60$ & $61-65$ & $66-70$ & $71-75$ \\
\hline & 2,3 & 5,1 & 0 & 2 & 1 & 1 \\
\end{tabular}

TABLE II.-Age Incidence: Females

\begin{tabular}{|c|c|c|c|c|c|c|c|c|c|c|c|}
\hline & \multicolumn{11}{|c|}{ Age Groups } \\
\hline & $25-30$ & $31-35$ & - $36-40$ & $41-45$ & $46-50$ & $51-55$ & $56-60$ & $61-65$ & $66-70$ & $71-75$ & $76-80$ \\
\hline $\begin{array}{l}\text { No. in each group } \\
\text { Duration of illness (in years) of each patient }\end{array}$ & $1 / 52$ & $\begin{array}{l}3 \\
8,7,7\end{array}$ & $\begin{array}{l}1 \\
5\end{array}$ & 0 & $\begin{array}{r}1 \\
20\end{array}$ & $18,35,6,2$ & 8,2 & 10 & $3,5,4,9$ & $31,3 / 12,4$ & 4,10 \\
\hline
\end{tabular}

$(65.7 \%)$ the lesions were considered to be indistinguishable from those of acute rheumatism, and of a non-specific infective nature in the remaining 8 . An active rheumatic process was present in 6 . There was a history of rheumatic fever in 3 only, and of probable rheumatic fever in 2 . From this extremely high incidence of the rheumatic type of heart disease in rheumatoid arthritis they concluded that there is an extremely close aetiological relationship between the two diseases.

An editorial comment (British Medical Journal, 1945, 1, 636) on Young and Schwedel's paper notes that the incidence of disease on the several valves was similar to that of acute rheumatism but that the incidence of mitral stenosis was not
The duration of disease is that given by the patient. No doubt there is considerable inaccuracy here. For example, in two patients who gave histories of only a few weeks' duration bony changes were apparent on $x$-ray examination. Accepting the figures as given by the patients, however, the average duration of the illness for the males was 3.2 years and the females 9.0 years-long enough for the development of any lesions.

That males were, on the average, admitted to hospital for treatment so much earlier than females is probably accounted for by the fact that in the breadwinner impairment of manual dexterity or locomotion is likely to be of more serious consequence than similar disabilities in the housewife. 
The cardiac rhythm was regular in all cases. The first mitral sound was split in 2 cases, and the intensity of this sound was diminished in 10 others, compatible with their age and varying degrees of emphysema ; otherwise no abnormality was noted. However, it would be only in the incidence and character of murmurs indicative of valvular disease that a parallel between this disease and rheumatic fever could be drawn. A particularly careful search was made for evidence of early mitral stenosis, but none was found. In 8 cases a faint systolic murmur was heard at the mitral area ; in all of these it was sharply localized. None of them was associated with a loud sharp first sound or with an accentuated second pulmonary sound. They were considered to be functional in origin. In one case only was there a loud mitral systolic murmur; it did not replace the first sound but was conducted well into the axilla. This occurred in a woman of 68 who had had rheumatoid arthritis for nine years, and the signs were thought to indicate organic mitral valve disease.

\section{Electrocardiographic Findings}

There are, of course, no electrocardiographic abnormalities pathognomonic of rheumatic fever; the study of electrocardiograms in rheumatoid arthritis is therefore of limited value in studying the aetiological relationship between the two diseases. According to Katz (1941) chronic rheumatic heart disease may result in tracings showing preponderance of one or other ventricle resulting from valve deformities, or the appearance of large, broad, or notched $P$ waves associated with auricular hypertrophy. Specific contour changes and arrhythmias may develop, such as frequent auricular premature beats, which are said to be particularly significant when they arise from multiple foci. Electrocardiograms were taken from 29 patients. Left axis shift was present in 17 ; there was a considerable incidence of slurring of the QRS complexes, and the $T$ wave was inverted in one case in Lead $I$, in another in Lead II, and in a third in Lead $\mathrm{CF}_{4}$. These abnormalities were consistent with the senile degeneration of the coronary arteries of old people, who formed the majority of the patients in this series. None of the records showed abnormal ventricular preponderance, the $\mathbf{P}$ waves were well formed, and the cardiac rhythm was unaffected. A woman of 71 , who gave a history of only three months' illness but who had obvious joint changes on $x$-ray examination, showed very low complexes in all leads when the first electrocardiogram was taken. Serial tracings showed an increasing amplitude of the complexes until five weeks later the voltage was well within normal limits. This suggests that there was transient myocardial damage during the acute phase of the illness, but it does not necessarily denote any common aetiological factor between rheumatoid arthritis and rheumatic fever; such findings might be encountered in many acute illnesses and toxic states.

Only one patient in the present series came to necropsy. She was an elderly woman who died of cerebral thrombosis. The naked-eye appearances and histological findings in the heart revealed no evidence of rheumatic infection.

\section{Discussion}

The results of this investigation serve to emphasize once again the extraordinary discrepancies which exist in the opinions of different workers. No doubt this is largely the result of failure to lay down generally acceptable criteria for the clinical diagnosis of cardiac lesions in the early stages of disease. Another difficulty is that of excluding cardiac abnormalities which are purely incidental and usually the accompaniments of senile degenerative changes in the myocardium and coronary arteries. After making liberal allowances for these complicating factors, it would appear that clinical evidence of mitral valve disease was elicited in only one patient in a series of 33 cases of rheumatoid arthritis. The literature cited raises the possibility that the incidence of subclinical valvular disease associated with rheumatoid arthritis in the present group was much higher than this, but in the solitary case examined at necropsy no cardiac abnormalities were detected. A final opinion on the concept of latent or subclinical endocarditis must await further pathological studies, which will need to be sufficiently comprehensive; the difficulties in the way of obtaining these data in a disease which per se is rarely fatal need no emphasis.

\section{Summary}

Recent literature on the subject of the aetiological relationships between rheumatic fever and rheumatoid arthritis is reviewed.

Among 33 patients suffering from rheumatoid arthritis only one was found to have a cardiac lesion which might have been attributable to the disease. Another patient came to necropsy, and was found to have neither histological nor naked-eye abnormalities of the heart.

A brief reference is made to the limited value of comparisons between the clinical manifestations of these two conditions in the present state of our knowledge of the rheumatic diseases.

I have pleasure in expressing my indebtedness to Prof. Noah Morris and Dr. Stanley Alstead, who assisted me in the preparation of this paper. My thanks are also due to Drs. Briggs and Cunningham, medical superintendents of Stobhill Hospital and the Eastern District Hospital, respectively, for permission to publish.

REFERENCES

Baggenstoss, A. H., and Rosenberg, E. F. (1941). Arch. intern. Med., 67, 241. (1944). Arch. Path., 37, 54.

B. (1943). Amer. J. med. Sci. 205, 42

Bennett, G. A., Zeller, J. W., and Bauer, W. (1940), Arch. Path., 30, 70.

Collins, D. H. (1937), J, Path. Bact. 45, 97.

Dawson, M. H., and Tyson, T. L. (1936). J. Lab. clin. Med., 21, 575.

Fraser T. N (1945). Ann. rheum. Dis 4, 71.

(1941) Clinical Electrocardiography,

(1932).J.Amer. med Ass, 98, 881 .

Sclater, J. G. (1943). Ann. rheum. Dis., 3, 195.

White, P. D. (1944). 'Heart Disease, New York.

Young, D., and Schwedel, J. B. (1944). Amer. Heart J., 28, 1.

\section{}

\section{INCIDENCE OF YAWS AND OF VENEREAL DISEASES IN LANGO (UGANDA)}

BY

\section{J. HACKETT, M.D.}

Director, Wellcome Museum of Medical Science, London

During a study of the bone lesions of yaws in Lango the high incidence of that disease became apparent. Since a medical survey was not practical, nor was it necessitated by trypanosomiasis, other measures were sought to ascertain more precisely the frequency of yaws. At the same time the incidence of venereal diseases in Lango and, for comparison, in Ganda was assessed. Medical reports were examined, out-patient attendance records analysed, and a labour force and a sample of a gaol population were studied. These data form the basis of this paper.

The results of a survey for leprosy are contained in the annual report of the District Medical Officer of Lango for 1931. Of 24,249 children under 15 years of age, $963(3.9 \%)$ were suffering from secondary yaws. Of 152 cases of secondary yaws with bone lesions studied by me in 1937-9, $131(86 \%)$ were under 10 years of age. Active secondary yaws in children was observed in practically every Lango community, and chiefs frequently stressed its high incidence. The absence of yaws in infants before the age of walking was also noted.

\section{Survey of Out-patient Attendances, Lira and Masaka}

Lira (Lango district), about $3,600 \mathrm{ft}$. $(1,100 \mathrm{~m}$.) above sea level and.north of Kampala, is in a part of Uganda described as a "yaws area"-i.e., one in which yaws is common and syphilis rarely occurs. Masaka (Ganda Province), about 4,300 ft. $(1,300 \mathrm{~m}$.) above sea level and south-west of Kampala, is in a part regarded as a "syphilis area " and in which yaws is infrequent. This characteristic local distribution of the two diseases applies only to the indigenes. Masaka has been chosen rather than Kampala for comparison with Lira, since it is not so large a centre as Kampala, and data from it are thus more likely to give an accurate picture of the conditions present in a rural community. The figures in Tables I, II, and IV were obtained from the monthly sick returns for Lira and Masaka, which were based mainly upon the diagnoses of trained African medical orderlies. Their diagnoses of the conditions concerned may be regarded as fairly accurate. Orderlies with experience in both syphilis and yaws areas were working in both clinics. 